\title{
Advances in imaging for lung emphysema
}

\author{
Katharina Martini, Thomas Frauenfelder \\ Institute of Diagnostic and Interventional Radiology, University Hospital Zurich, Zurich, Switzerland \\ Contributions: (I) Conception and design: T Frauenfelder; (II) Administrative support: All authors; (III) Provision of study materials or patients: All \\ authors; (IV) Collection and assembly of data: All authors; (V) Data analysis and interpretation: All authors; (VI) Manuscript writing: All authors; (VII) \\ Final approval of manuscript: All authors. \\ Correspondence to: Prof. Thomas Frauenfelder. Diagnostic and Interventional Radiology, University Hospital Zurich, Rämistrasse 100, 8091 Zurich \\ (CH), Switzerland. Email: Thomas.frauenfelder@usz.ch.
}

\begin{abstract}
Lung emphysema represents a major public health burden and still accounts for five percent of all deaths worldwide. Hence, it is essential to further understand this disease in order to develop effective diagnostic and therapeutic strategies. Lung emphysema is an irreversible enlargement of the airways distal to the terminal bronchi (i.e., the alveoli) due to the destruction of the alveolar walls. The two most important causes of emphysema are (I) smoking and (II) $\alpha 1$-antitrypsin-deficiency. In the former lung emphysema is predominant in the upper lung parts, the latter is characterized by a predominance in the basal areas of the lungs. Since quantification and evaluation of the distribution of lung emphysema is crucial in treatment planning, imaging plays a central role. Imaging modalities in lung emphysema are manifold: computed tomography (CT) imaging is nowadays the gold standard. However, emerging imaging techniques like dynamic or functional magnetic resonance imaging (MRI), scintigraphy and lately also the implementation of radiomics and artificial intelligence are more and more diffused in the evaluation, diagnosis and quantification of lung emphysema. The aim of this review is to shortly present the different subtypes of lung emphysema, to give an overview on prediction and risk assessment in emphysematous disease and to discuss not only the traditional, but also the new imaging techniques for diagnosis, quantification and evaluation of lung emphysema.
\end{abstract}

Keywords: Emphysema; imaging; chronic obstructive pulmonary disease (COPD)

Submitted Jan 07, 2020. Accepted for publication Apr 06, 2020.

doi: $10.21037 /$ atm.2020.04.44

View this article at: http://dx.doi.org/10.21037/atm.2020.04.44

\section{Introduction}

Lung emphysema represents a major public health burden and still accounts for five percent of all deaths worldwide (1). Hence, it is essential to further understand this disease in order to develop effective diagnostic and therapeutic strategies.

Lung emphysema is an irreversible destruction of alveolar walls resulting in enlargement of the distal airspaces $(2,3)$. Although emphysema initially does not involve fibrosis, end-stage emphysema may lead to a reparative response that ultimately leads to pulmonary fibrosis. There are three different types of pulmonary emphysema: (I) panlobular, (II) centrilobular, and (III) septal emphysema
$(3,4)$. Additionally, severity and distribution of lung emphysema can give hints to causes of emphysema: While for example smoking related emphysema is typically predominant in the upper lung areas, lung emphysema due to $\alpha 1$-antitrypsin-deficiency is located in the basal lung areas. Lung emphysema distribution can also provide important information for the pre-operative evaluation of patients: heterogeneous emphysema is known to be more responsive to lung volume reduction (LVR) as for example homogeneously distributed emphysema (5).

This knowledge makes imaging a central player in the evaluation of lung emphysema: The main goal of imaging is to assess the extent and distribution of emphysema and to detect concomitant findings such as infections or identify 

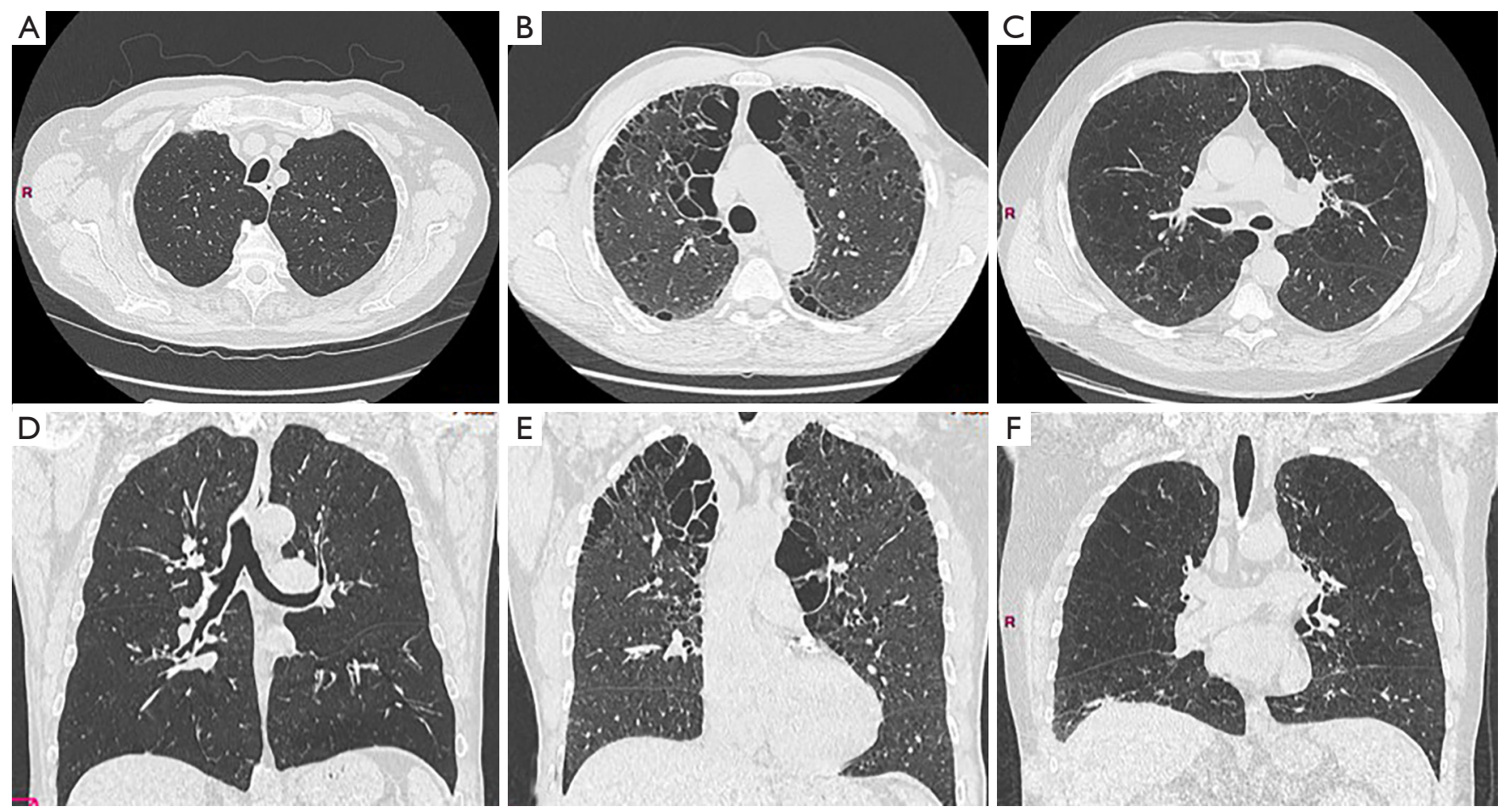

Figure 1 Different types of emphysema in axial (upper row) and coronal (lower row) reconstructions. (A,D) mild form of centrilobular emphysema, (B,E) paraseptal emphysema, and (C,F) panlobular emphysema.

malignancies (6).

The imaging modalities, which can be used for the evaluation of emphysema, are manifold: Due to its high spatial resolution, computed tomography (CT) remains the gold standard in the evaluation of lung parenchymabut other imaging modalities, such as magnetic resonance imaging (MRI) or scintigraphy, have increased its importance in the last years.

The aim of this review article is to present the different subtypes of lung emphysema, to give an overview on prediction and risk assessment in emphysematous disease and to discuss not only the traditional, but also the new imaging techniques for diagnosis, quantification and evaluation of lung emphysema.

\section{Types of emphysema}

Elastase is produced by alveolar macrophages and neutrophils, both of which are increased in smokers. Elastase is a powerful destructive enzyme, which functions in the host defence mechanism, but an excess can be harmful to the tissues. $\alpha 1$-antitrypsin normally neutralizes elastase. Either a surplus of elastase (in smoking-related emphysema) or a deficiency of $\alpha 1$-antitrypsin (in $\alpha 1$ antitrypsin-deficiency) can result in lung destruction and finally in emphysema $(2,3)$.

Lung emphysema can be divided into three major subtypes at autopsy: (I) centrilobular, (II) paraseptal, and (III) panlobular emphysema. These subtypes can also be defined visually on CT (Figure 1).

\section{Centrilobular emphysema (CLE)}

CLE is generally described as an abnormal magnification of airspaces centered on the respiratory bronchiole with fusion of destroyed lobules in grievous cases $(3,7,8)$. CLE is a smoking-related disease of the lungs and predominantly affects the upper lobes. As in all smoking-related lung diseases (such us respiratory bronchiolitis, desquamative interstitial pneumonia, Langerhans Cell Histiocytosis of the lung) also in lung emphysema the center of the secondary pulmonary lobule is primarily affected. Moreover, single subtypes of emphysema may have different pathophysiology. For instance, Smith et al. (9) could show that tobacco users with mainly CLE had a more important level of cigarette exposure, higher lung volumes, and lower lung diffusion 

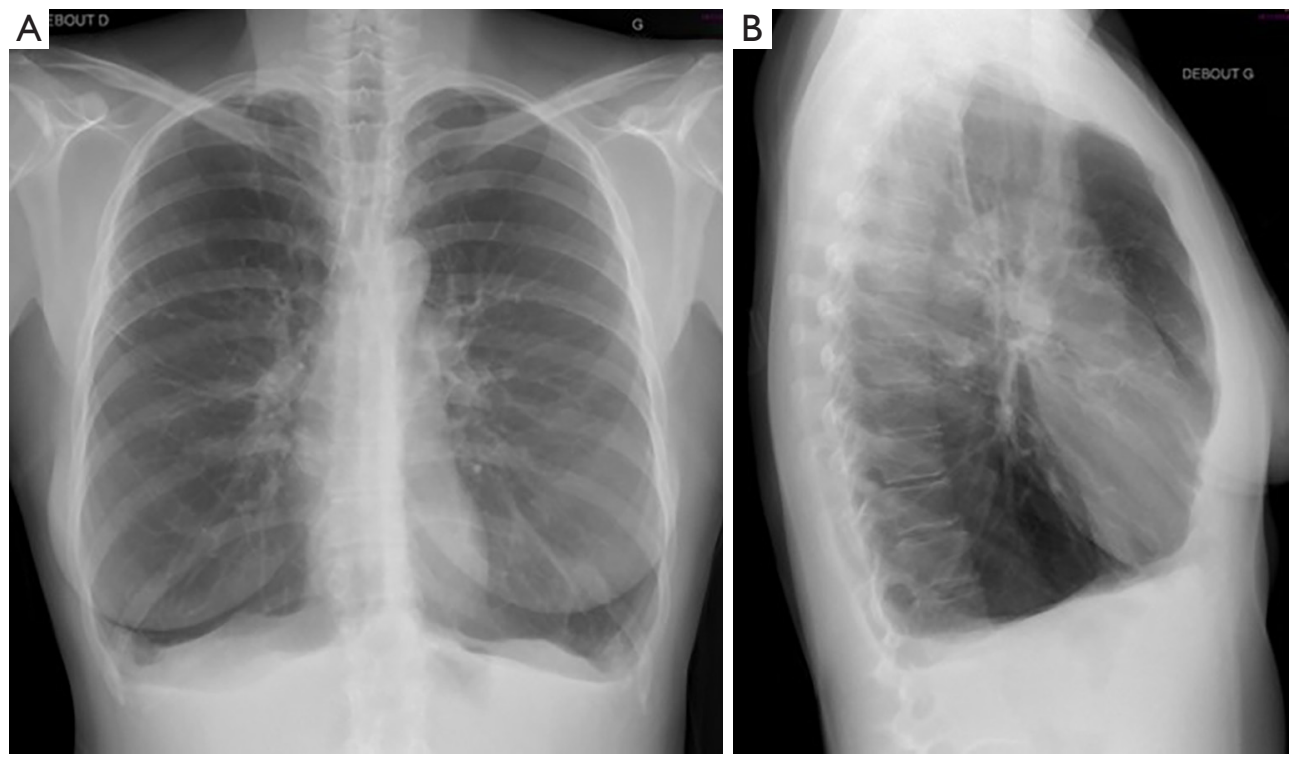

Figure 2 Conventional chest radiography of a 38-year-old female patient with $\alpha 1$-antitrypsin deficiency showing typical signs of emphysema: (I) the barrel shaped thoracic cage with flattening of the hemi-diaphragmatic domes, (II) hyperlucency of the lung tissue due to overinflation and rarefication of vessels, (III) enlargement of the retrosternal clear space $>2.5 \mathrm{~cm}$; and (V) narrow configuration of the cardiac silhouette.

capacity than those lacking emphysematous changes of the lung. Contrarily, smokers with a predominantly "panlobular" pattern of emphysema had a comparably lower body mass index than smokers without emphysematous changes (10).

\section{Paraseptal emphysema}

Paraseptal emphysema allude to emphysematous lung changes adjacent to the pleura $(3,7,8)$. It is usually seen in combination with other forms of emphysema (10) and also smoking related. Especially in patients who smoke marijuana severe cases of paraseptal emphysema are seen (11). Due to its subpleural location, paraseptal emphysema is a risk factor for pneumothorax (10).

\section{Panacinar emphysema}

Panacinar emphysema (or also referred to as panlobular emphysema) is often characterized as abnormal dilation distributed throughout the pulmonary lobule. It affects the entire acinus diffusely throughout the lung. $\alpha 1$ antitrypsin-deficiency is cause of panlobular emphysema. The emphysematous changes are usually more sever at the lung bases (12). Another cause of panacinar emphysema is primary ciliary dysfunction.
Additionally to the different subtypes of emphysema, severity and distribution can be evaluated on CT and can give important hints to the causes of emphysema: While for example smoking related emphysema is typically predominant in the upper lung areas, lung emphysema due to $\alpha 1$-antitrypsin-deficiency is located in the basal lung areas $(3,7,8)$.

\section{Traditional imaging in emphysema}

\section{Conventional radiography}

Conventional chest radiography (chest $\mathrm{X}$-ray) has been the backbone of imaging for more than 100 years (13). Although chest X-ray is one of the oldest and least sophisticated imaging examinations, it remains one of the most commonly used methods due to its wide availability, low cost, and fast implementation (14-17). The two most important signs of lung emphysema on X-ray are (I) the barrel shaped thoracic cage with flattening of the hemi-diaphragmatic domes, and (II) hyperlucency of the lung tissue due to overinflation and rarefication of vessels (Figure 2). Auxiliary findings comprise enlargement of the intercostal areas, enlargement of the retrosternal clear space $>2.5 \mathrm{~cm}$, narrow configuration of the cardiac silhouette, increased markings pattern, and the presence of bullae (4). 
Since, conventional radiography is a projection-based imaging technique, it is often impossible to distinguishing structures of equal density next to each other or overlapping different structures (18). Chest X-ray has only a sensitivity of $40 \%$ in identifying emphysema (4), little specificity and high interobserver disagreement. These facts hinder a solid and reproducible quantification of emphysema burden in COPD patients (19).

The main role of $\mathrm{X}$-ray in emphysema is to investigate for concomitant findings that should be evaluated before therapy planning (infection, pleural scarring and pulmonary masses) and plays an essential role in follow-up imaging. Chest X-ray is broadly used for postinterventional evaluation following placement of endobronchial valve, sealant or coil implantation or after LVR and its potential complications such as device displacement or pneumothorax.

For a better representation of the extent and distribution of emphysematous lung changes, as well as for a sensitive recognition of absolute contraindication for LVR procedure, further evaluation with chest CT is advised.

\section{$C T$}

Due to its high spatial resolution, the opportunity of three-dimensional reformation and quantification CT is the preferred imaging modality in the evaluation of lung emphysema. CT allows for an easy quantification and characterization of emphysematous destructed lung $(19,20)$. CT is also the imaging modality of choice when it is up to assess heterogeneity and distribution of emphysematous lung changes when it is up to find suitable candidates for LVR or endobronchial valve placement: The National Emphysema Treatment Trial (NETT) for example, revealed that subjects with upper lobe predominant or heterogeneous emphysematous changes are benefitting the most from LVR (5), while patients with a homogeneous distribution had a lesser beneficial effect after treatment.

$\mathrm{CT}$ is also used to identify concomitant conditions such as malignancy and airway involvement (i.e., bronchiectasis, tracheomalacia, lung compression, mucus plugging and thickening of the bronchial walls) (19). Moreover, with $\mathrm{CT}$ it is possible to detect the presence of air trapping and bronchial wall collapse, when additional expiratory CT series are acquired (4).

\section{Quantification of emphysema}

Quantification can be done by (I) visual scoring, (II) semiquantitative methods using densitometry or by (III) quantification.

The most widespread utilized visual score is the Goddard score, through which the observer evaluates the degree of severity of emphysematous lung changes at three distinct zones in either individual lung. For each of these six zones, a numerical score of 0 to 4 is given ( 0 is defined as no presence of emphysema to 4 which is defined as more than $76 \%$ of emphysema). The Goddard score can range from 0 to 24 . Furthermore, the emphysema distribution (i.e., heterogeneity) is also assessed at each location. Different studies showed a high agreement between readers, when scoring is performed by experienced radiologists or pulmonologists $(21,22)$. Contrary to this, other authors still report high interobserver variability of this technique: In the NETT, despite the use of experienced and previously trained chest radiologists, significant inter- and intra-reader variability in the quantification of lung emphysema was reported (5).

Due to the fact that visual scoring is neither sensitive nor precise, to date primarily semiquantitative and quantitative approaches are used for the evaluation of the extend of lung emphysema.

Semiquantitative evaluation uses simple methods which do not require the use of sophisticated software: for instance, by (I) sagittal reformations, and (II) colour coding the severity and distribution of emphysema using the CT densitometry can be performed. Further, CT densitometry images enable for a precise, less observer-dependent evaluation of emphysematous lung changes compared to axial and multiplanar CT images and have therefore potential to be used in LVR surgery planning (23).

To date, quantitative methods are the gold standard in the assessment of and manifold commercially available software tools allow for an automated quantification of lung emphysema. The first step is to virtually remove other anatomic structures such as the chest wall and the mediastinum from the lung parenchyma. In a second step, the remaining lung parenchyma is evaluated voxel by voxel for different CT attenuations, the so-called Hounsfield Units (HU). HU are used to measure the density of each voxel and according to generally accepted thresholds [Gevenois et al. $(24,25)$ recommended a cut-off value for lung emphysema on CT scans is $-950 \mathrm{HU}]$ it is possible to distinguish normal lung tissue from emphysema $(19,26)$. Finally, the burden of emphysema is defined as the relative amount of lung parenchyma inferior to the chosen threshold. Both, the observer and the software based 
method, can be used additionally for the classification of the anatomic distribution of emphysematous lung changes (6). Newer and more sophisticated techniques allow for a depiction of emphysema clusters, providing more profound information on heterogeneity (27). Despite the above mentioned semi-quantitative and quantitative approaches, the validation of the source images remains crucial in order to don't overlook concomitant findings such as malignancies, infections or evaluation of fissures.

\section{Fissure evaluation}

When patients are evaluated for endovascular valves placement as possible treatment options, lung fissure evaluation plays besides the quantification and distribution of emphysema a crucial role (28). Sciurba et al. evaluated patients were HRCT was used to evaluate distribution of emphysema and fissure anatomy to find suitable lung regions for endobronchial valve placements and could show that these patients had a better post-interventional outcome than the control-group (29). This is due to the fact, that incomplete fissures contribute to collateral ventilation and hamper the results of endobronchial valve treatment.

In a large European study on endobronchial valves lung volume reduction (EBV) complete interlobar fissures were found only in one-third of patients (30).

Nowadays, there are to options for the assessment of fissure integrity: (I) invasively with the Chartis Pulmonary assessment System (CPAS) (31) or (II) non-invasively by using CT. Quantitative CT has been shown to be comparable to CPAS and is therefore a useful, non-invasive method to select suitable candidates for EBV (32). When fissure integrity quantified by CT is below $95 \%$, additional bronchoscopic assessment with CPAS is necessary to exclude alternative ventilation.

One drawback in CT based evaluation is that fissure validation can be difficult in cases with severe emphysema, showing low interobserver agreement (33). In these cases multiplanar reformations in coronal and sagittal planes appeared to be useful in the assessment of fissure integrity (34).

\section{Minimum intensity projection (MinIP)}

An easy and straightforward method for a better visualization of pulmonary emphysema is the MinIP: MinIP images highlight areas with reduced CT attenuation in the lung parenchyma. This allows prompt detection of emphysematous areas of the lung (35). Ghonge et al. suggests window widths of 350-500 $\mathrm{HU}$ and a window level of -750 to $-900 \mathrm{HU}$ for image interpretation.

\section{Scintigraphy}

A different imaging modality, which can be used in the evaluation of severe lung emphysema is scintigraphy (Figure 3). With scintigraphy it is possible to visualize ventilation and perfusion of the lungs. With this method upper-lobe predominance and heterogeneity of emphysematous lung changes can be visualized and combined with functional data such as perfusion. The technique is especially helpful in the evaluation for the detection of severely emphysema affected areas in the lung in heterogeneous emphysema, but struggles in subjects with a homogeneous distribution of emphysema (36). In scintigraphy the lung is divided in three distinct zones (i.e., upper, middle and lower zonr). Information on tracer uptake, corresponding to ventilation and perfusion, are provided for these three distinct lung zones.

Some authors, despite the high agreement between lung perfusion evaluated on HRCT and on scintigraphy, criticize scintigraphy for the lack of additional information to HRCT making scintigraphy superfluous in the assessment of lung emphysema $(37,38)$.

\section{New techniques:}

\section{Dual energy $C T$}

\section{Conventional dual energy CT}

Another technique for quantification of emphysema is based on dual-energy CT (DECT) (Figure 4). With this technique pulmonary perfusion is assessed by the measurement of iodine dispersion maps or lung ventilation by measurement of the distribution of injected iodine contrast media (39). The principle the technique is the acquisition of images at different $\mathrm{X}$-ray energies in order to analyse the resultant images for differences in attenuation. With this approach iodine maps of the lung parenchyma reflecting the perfusion can be generated. These maps can provide additional physiological information in patients with lung emphysema as mere morphological assessment with standard CT can (40). Pansini et al. (41) evaluated pulmonary perfusion on a lobar level in tobacco users with dual-energy CT to investigate for connections between the degree of parenchymal destruction and alteration of pulmonary perfusion. They found that regional alterations 

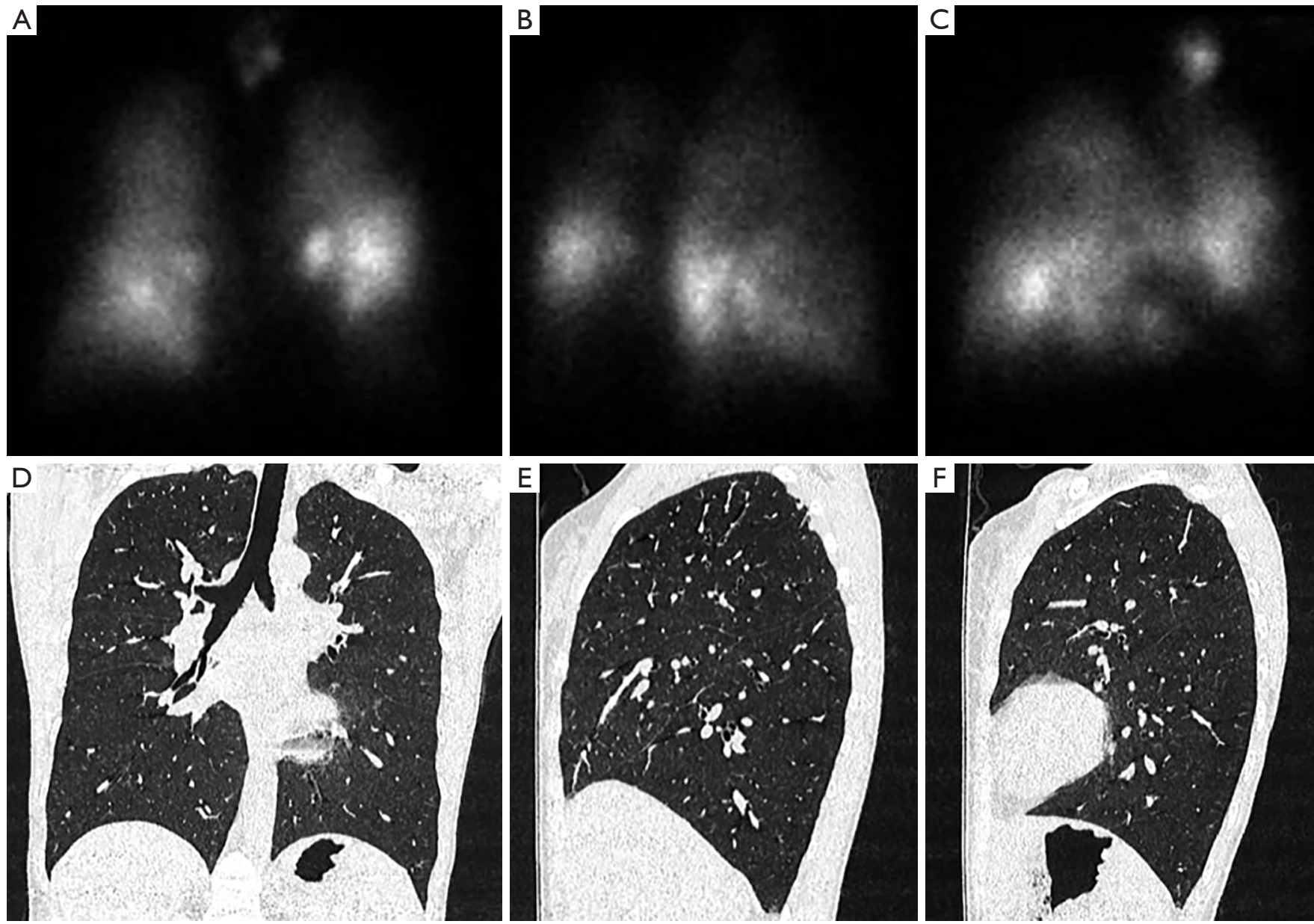

Figure 3 Lung scintigraphy in a 43-year-old female patient with COPD GOLD IV showing perfusion maps in (A) anteroposterior, (B) right oblique and (C) left oblique perfusion maps. Emphysematous lung areas in CT lung windows (D,E,F) correspond to areas on scintigraphy with reduced or no tracer uptake.

of pulmonary perfusion can be visualized by dual-energy $\mathrm{CT}$ in smokers with predominant emphysematous lung changes.

Lung perfusion scintigraphy has been traditionally used to assess potential candidates for LVR surgery with severe emphysema. Despite this method can assess the perfusion pattern in patients with severe emphysematous changes, it suffers from low spatial resolution. MRI seems to be a more promising technique, since it has shown to have high diagnostic accuracy in the detection of perfusion abnormalities and the ability to provide lobar and segmental assessment of perfusion defects $(42,43)$. Yet, none of the afore mentioned methods is able to evaluate morphological changes of the lung parenchyma, such as thin-collimated high-resolution CT can. Hence, full assessment of lung destruction and impaired lung function require a combination of morphological and functional information or creation of fusion images using an additional technique. This is in contrast to dual-energy CT:

The technique can be used as a one-stop-shop tool, which is able to combine the functional information of scintigraphy/MRI with the superior structural information of CT.

\section{Xenon enhanced dual energy CT}

A novel technique for the quantification of emphysematous lung changes poses Xenon enhanced dual energy CT (XEDECT). Xenon is a stable, nobel gas, occurring naturally in the atmosphere at a concentration of $0.05 \%$. Its atomic 

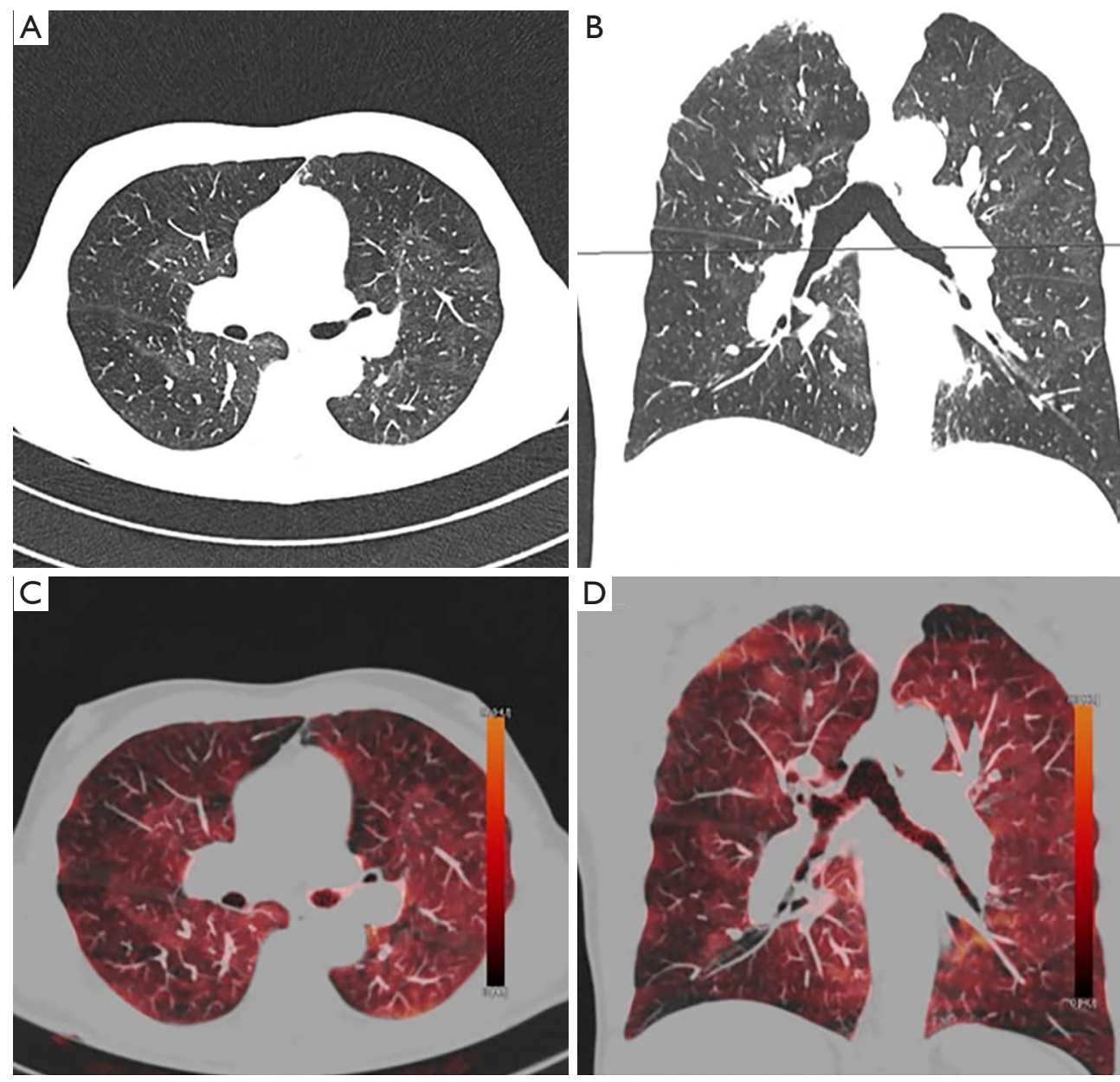

Figure 4 Showing the distribution of emphysema in a 64-year-old male patient with COPD on (A,B) computed tomography images in axial and coronal slices as well as (C,D) dual-energy CT (DECT) maps superimposed to CT lung window images.

number is similar to that of iodine (44).

Different trials could show that lung areas enhanced by xenon strongly correlate with obstructive ventilation impairment (45). Further, ventilation defects depicted on Xe-DECT correlate significantly with airflow obstruction on lung function tests (46-48). This image information is utilized for the depiction emphysema distribution and localization and can give important hints which patients might be suitable for treatment. There is evidence, that a changes of lung parenchyma among inspiration and expiration CTs and xenon dynamic change correlate significantly, and might be more robust than lung function parameters except FEV1 (46). Additionally, XE-DECT showed in an animal model that the technique allows the quantification of collateral ventilation and detection of differences between canine and swine models of bronchial obstruction (49). In a study evaluating 1830 CT cerebral blood flow examinations using XE-DECT with $32 \%$ inhaled stable oxygen was used the following adverse events were reported: $3.6 \%$ occurrence of respiratory rate delay greater than 10 seconds, $0.4 \%$ cephalgia, $0.2 \%$ seizures, $0.2 \%$ nausea and vomiting, and $0.1 \%$ change in neurologic status (44).

Another important drawback of XE-DECT is that Xenon is not readily available in all scanning sites; and images have to be acquired in specified centres.

\section{MRI (morphologic, functional and dynamic)}

\section{Morphologic MRI}

MRI of the lung is the newest imaging technique, which has been established for the evaluation of lung parenchyma, 

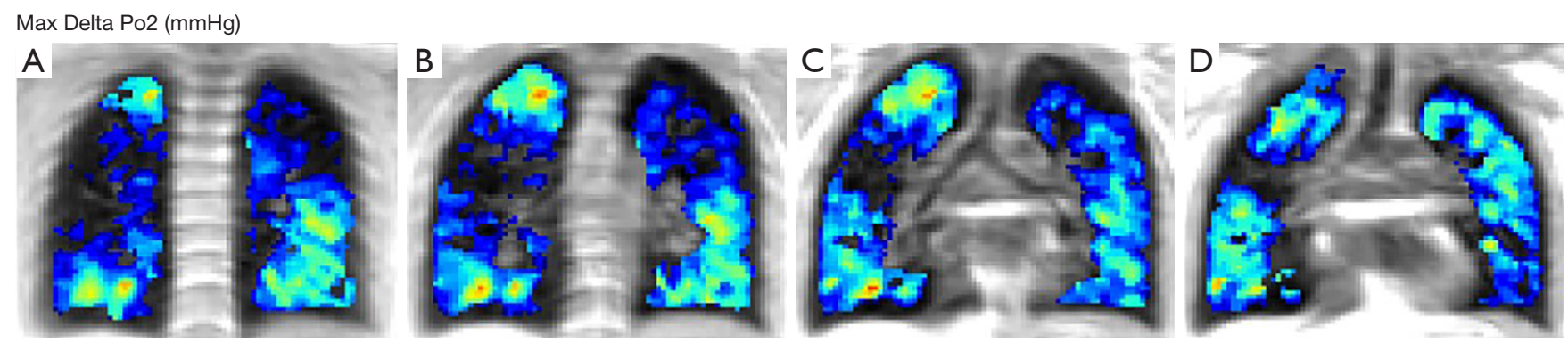

Figure 5 Subtraction maps of oxygen enhanced MRI in a patient with chronic obstructive pulmonary disease showing areas of emphysema in the upper lobes and middle lobe with lower mean $\Delta \mathrm{O}_{2}$ pressure uptake of oxygen compared to the less emphysematous lung parts in the lower lobes.

and especially emphysema. This is due to the comparable low spatial resolution of MRI and that the technique struggles in the lung with the lack of hydrogen ions, which are essential for image generation in MRI. While so called "plus pathologies" (such as consolidation, atelectasis, mucus plugging) can be depicted easier on MRI, the "minus pathologies" (such as bullae and emphysema) are more difficult to capture. Yet, due to the development of novel sequences, the role of MR in lung imaging is changing and the technique has achieved a more important role in the evaluation of lung parenchyma. Ultrashort and Zero Echo-time sequences are nowadays able to capture lung parenchyma pathologies almost with the same sensitivity as CT does (50-52). Additionally, contrast-enhanced MRI, similar to DECT is a noninvasive technique and can be used to repeatedly assess clinicopathological severity. The use of contrast enhanced MRI allows for obtaining information on pulmonary vasculature, and the quantification of the relative pulmonary blood (53).

\section{Functional MRI using oxygen or noble gases}

Despite CT might still be superior in the visualization of subtle lung parenchyma changes, the power of MRI is functional imaging $(54,55)$. Not only lung morphology but also lung function can be assessed using pure oxygen or noble gases: Hyperpolarized noble gases or molecular oxygen function as inhaled MRI contrast agents which are able to show regional ventilation and perfusion without delivering radiation to the examined patient (56-58).

The depiction of oxygen distribution is of high physiological and pathophysiological interest, since oxygen transport represents the primary function of the lungs and not the distribution of non-physiological substances which are assessed in a nuclear medicinepulmonary function test (59). The difference in lung parenchymal signal intensities between inhaled room air and $100 \%$ oxygen is minimal, and visual representation is generally done by the generation of subtraction maps (Figure 5).

Ohno et al. (60) were one of the first to show the feasibility of dynamic oxygen-enhanced MRI in a clinical setting. They hypothesized that dynamic oxygen enhancement reflects the regional diffusing capacity of the lung.

Likewise, with the administration of intravenous contrast agents the signal of the lung during dynamic MRI can be post-processed by calculating the inflow over time to receive quantitative parameters for pulmonary blood flow, blood volume and mean transit time $(42,61)$. The quantitative maps of lung perfusion obtained by this technique are similar to those of perfusion scintigraphy $(62,63)$. Dynamic contrast-enhanced MRI has also been recommended for the evaluation and follow-up of COPD disease severity (64).

A different approach is Fourier decomposition lung MRI, a novel method for functional lung imaging without the use of contrast agents. With the method regional lung perfusion and ventilation-related information can be assessed simultaneously (65). The strength of the method is its short acquisition time of only 30 seconds, but the techniques suffers from a lack in robustness, resulting in too many artifacts (66).

Even though CT is potentially better in the depiction of subtle lung parenchyma changes, functional parameters evaluated with MRI allow for the depiction of ventilation and time resolved lung perfusion as well as breathing dynamic and functional imaging of the diaphragm.

\section{Dynamic MRI}

Beside the visualisation of morphologic changes as well as ventilation and time resolved lung perfusion, MRI is 

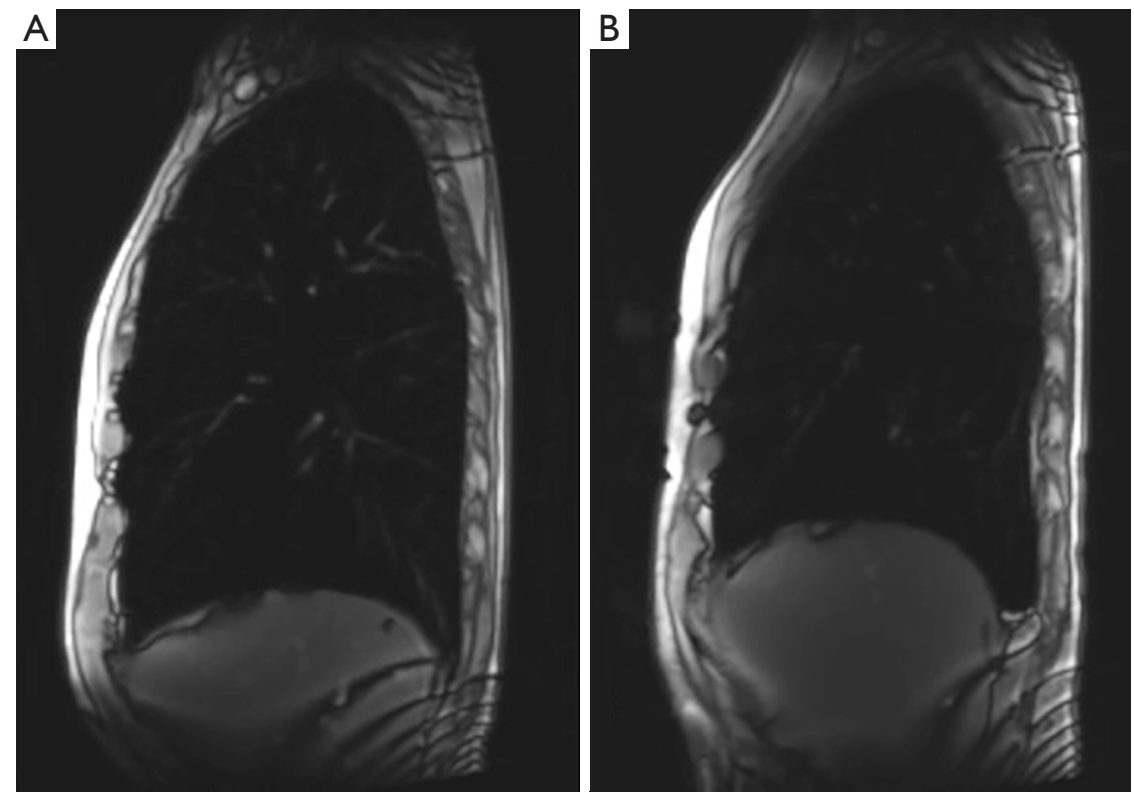

Figure 6 Images of 67-year-old male patients before (A) and after (B) undergoing lung volume reduction surgery. (A) resembles typical findings of emphysema such as flattening of the hemidiaphragmatic dome; postoperative image (B) shows a different configuration of the hemidiaphragmatic dome with a convex shape and lower lung volumes.

a well-recognized technique for the evaluation of lung volumes and respiratory mechanics: Apart from upperlobe predominance of lung emphysema and heterogeneity, MRI is able to capture impaired motion of the respiratory muscles inclusive the diaphragm, which are additional factors in chronic obstructive pulmonary disease. This is possible, because MRI is an imaging technique, which does not expose the patient to radiation, and image acquisitions can therefore be performed over a longer time. This gives the possibility to evaluate not only static images, but to investigate also for the dynamic and the function of respiration and respiratory muscles. Suga et al. (67) could demonstrate that with dynamic MRI it is possible to noninvasively visualize dynamic respiratory motions of the respiratory muscles. Martini et al. could further show, that the evaluation of breathing dynamic might be beneficial in treatment planning and selection of patients who might benefit the most from LVR (Figure 6) (68).

\section{Radiomics in emphysema}

Interpretation of medical images relies on visual assessment by an observer, mostly the radiologist. Gained knowledge of anatomical and physiological variations determines the recognition of patterns that are within normal ranges and allows identifying appearances outside these ranges to be categorized as pathologic (69). More and more, advancement in standardisation efforts, applications of analysis techniques to extract quantitative information. Radiomics, defined as the conversion of medical images to higher-dimensional data, is a novel research area: The technique has attracted increased attention in recent years and several studies could show that radiomics can be beneficial for prognosis and diagnosis of multiple diseases, especially malignancies (32-34). Feature extraction is a crucial step in radiomics and implies the computation of texture, density and shape features from predefined regions of interest (ROIs). The biggest advantage of radiomics is that with the technique it is possible to obtain an objective quantification of tissue characteristics and abnormalities in radiological images (16-19). Due to the high objectivity and reproducibility of data, radiomics shows great potential as support for clinical decision-making (20), since it is not hampered by subjective evaluation or experience of the radiologist.

To date, an amount of neural networks and statistical models are available to help radiologists and clinicians with objective and reproducible computer-based evaluation of lung parenchyma. For instance, CALIPER (Computer Aided Lung Informatics for Pathology Evaluation and 

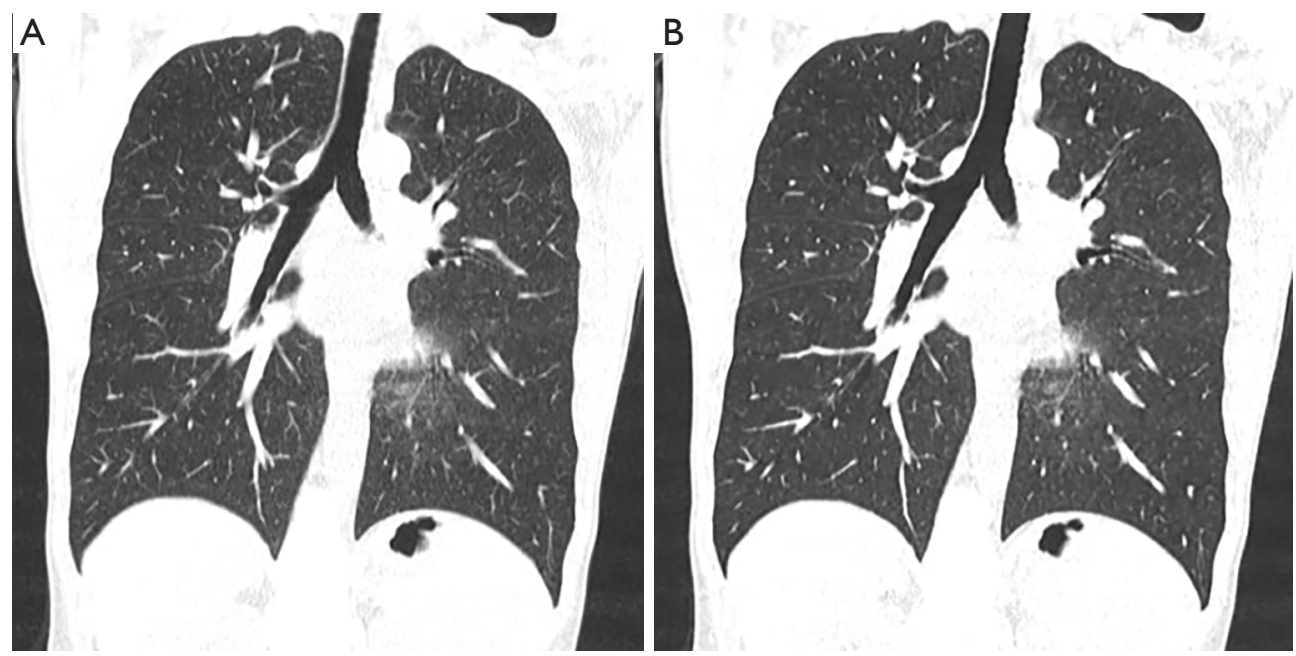

Figure 7 A 43-year-old female patient with upper lobe predominant centrilobular emphysema (A) reconstructed with filtered back projection (FBP) and (B) reconstructed with advanced modeled iterative reconstruction (ADMIRE) strength level 5.

Rating) developed at Mayo Clinic (Rochester, MN), is a digital platform for a near real-time qualitative and quantitative assessment of lung parenchyma patterns on CT (70,71).

Further, Occhipinti et al. could show that presence and severity of emphysematous lung changes in subjects with chronic obstructive pulmonary disease, as quantified by CT metrics and radiomics can be estimated by mathematical modeling of airway function obtained from standard lung function testing (72).

Quantitative imaging biomarkers have the potential to provide objective decision-support tools for patient the management (69). However, despite the promising results, radiomics is not yet implemented in the daily clinical routine, mostly due to its vulnerability in changes of scanning protocols: differences in scanning parameters such as type of CT scanner, tube voltage, tube current, reconstruction kernel and contrast agent may affect image texture and may hamper the functionality of an algorithm previously optimized for other scanning parameters. Before Radiomics and Artificial Intelligence can be applied, measurement variability needs to be understood and systems for data acquisition and analysis harmonised (69).

\section{Iterative reconstruction: how to overcome the changes in image texture}

In spite of the considerable advantages of quantitation, only a few quantitative imaging scores are used in clinical decision-making due to several obstacles: (I) Difficulties in the harmonization of data acquisition and analysis, (II) lack of international standards without routine quality assurance (QA) and quality control (QC) processes, (III) poor validation of quantitative biomarkers that are subject to errors in interpretation (69). For example, several trials indicate that automated quantification of emphysematous lung changes is highly reproducible, when identical scan parameters and reconstruction algorithms are used (5). Yet, different scanners and especially different reconstruction algorithms might hamper the reliable function of semiquantitative and quantitative approaches due to the different image appearance (Figure 7): different authors have already criticized iterative reconstruction (IR) techniques in visual approaches for the artificial texture of the reconstructed images and the appearance changes of images reconstructed with IR (73,74). Martini et al. (75), for example, found that the use of IR techniques was not beneficial for increasing sensitivity for the detection interstitial lung changes in reduced dose protocols, despite image noise was reduced.

Messerli et al. (76), who evaluated the impact of IR and reduced dose CT on quantitative emphysema evaluation, found that the mean lung attenuation of reduced dose CT was significantly altered by IR. In addition, the agreement of mean lung attenuation between standard dose and reduced dose CT with IR was poor; and the agreement between standard and reduced dose CT without the use of IR was substantial. These findings imply that for CT with 
reduced dose protocols or IR an adaptation of the generally accepted and recommended thresholds for HU values $(24,25)$ is necessary in order to distinguish emphysematous lung parenchyma from normal lung tissue. Since the agreement between standard and reduced dose CT without the use of IR was substantial, a different approach could be applied: for mere quantification, images not post-processed with IR algorithms could be used, while using the post processed only for the visual assessment.

\section{Risk assessment in patients with emphysema}

Different studies could show, that lung emphysema detected on CT is associated with important clinical outcomes, such as mortality (77-80). Quantitative assessed emphysematous lung changes are also associated with increased all-cause mortality in subjects with chronic obstructive pulmonary disease (20). Lynch et al. Found that the visual presence and severity of emphysema is associated with significantly increased mortality risk, independent of the quantitative severity of emphysema (20). Smith et al. reported in a systematic literature review that lung emphysema visually detected on CT is linked with increased incidence of lung cancer. Interestingly, this association did not hold true for automated emphysema detection (78): The two largest prospective studies derived from screening trials after adjustment for smoking history and airflow obstruction, reported a significant risk of lung cancer in patients were emphysema was visually detected on chest CT $(81,82)$. In contrast to this, two case-control studies were the presence of lung emphysema was assessed with quantitative approach, no association of emphysema with lung cancer could be found $(83,84)$. The reason for these results might be that quantitative or semiquantitative approaches are more sensitive in the detection of also subtle emphysema, which overall has lower impact on malignancy development.

A different approach was to evaluate different emphysema distributions and their clinical relevance (9): in this study centrilobular and panlobular emphysematous lung changes were linked with more severe symptoms, and reduced exercise capacity independent of airflow obstruction. Contrary to this, paraseptal emphysema was only of little physiologic significance. Lung emphysema was also seen in a substantial minority of subjects without chronic obstructive pulmonary disease with functional impairment.

\section{Acknowledgments}

Funding: None.

\section{Footnote}

Provenance and Peer Review: This article was commissioned by the Guest Editor (Bassam Redwan) for the series "Lung Emphysema" published in Annals of Translational Medicine. The article was sent for external peer review organized by the Guest Editor and the editorial office.

Conflicts of Interest: Both authors have completed the ICMJE uniform disclosure form (available at http://dx.doi. org/10.21037/atm.2020.04.44). The authors have no conflicts of interest to declare.

Ethical Statement: The authors are accountable for all aspects of the work in ensuring that questions related to the accuracy or integrity of any part of the work are appropriately investigated and resolved.

Open Access Statement: This is an Open Access article distributed in accordance with the Creative Commons Attribution-NonCommercial-NoDerivs 4.0 International License (CC BY-NC-ND 4.0), which permits the noncommercial replication and distribution of the article with the strict proviso that no changes or edits are made and the original work is properly cited (including links to both the formal publication through the relevant DOI and the license). See: https://creativecommons.org/licenses/by-nc-nd/4.0/.

\section{References}

1. Hoyert DL, Xu J. Deaths: preliminary data for 2011. Natl Vital Stat Rep 2012;61:1-51.

2. Celli BR, MacNee W. Standards for the diagnosis and treatment of patients with COPD: a summary of the ATS/ ERS position paper. Eur Respir J 2004;23:932-46.

3. The definition of emphysema. Report of a National Heart, Lung, and Blood Institute, Division of Lung Diseases workshop. Am Rev Respir Dis 1985;132:182-5.

4. Thurlbeck WM, Simon G. Radiographic appearance of the chest in emphysema. AJR Am J Roentgenol 1978;130:429-40.

5. Fishman A, Martinez F, Naunheim K, et al. A randomized 
trial comparing lung-volume-reduction surgery with medical therapy for severe emphysema. N Engl J Med 2003;348:2059-73.

6. Washko GR, Hoffman E, Reilly JJ. Radiographic Evaluation of the Potential Lung Volume Reduction Surgery Candidate. Proc Am Thorac Soc 2008;5:421-6.

7. Leopold JG, Gough J. The centrilobular form of hypertrophic emphysema and its relation to chronic bronchitis. Thorax 1957;12:219-35.

8. Hansell DM, Bankier AA, MacMahon H, et al. Fleischner Society: glossary of terms for thoracic imaging. Radiology 2008;246:697-722.

9. Smith BM, Austin JH, Newell JD, et al. Pulmonary emphysema subtypes on computed tomography: the MESA COPD study. Am J Med 2014;127:94.e7-23.

10. González J, Henschke CI, Yankelevitz DF, et al. Emphysema phenotypes and lung cancer risk. PloS one 2019;14:e0219187.

11. Tashkin DP, Roth MD. Pulmonary effects of inhaled cannabis smoke. Am J Drug Alcohol Abuse 2019;45:596-609.

12. Stoller JK, Aboussouan LS. A review of alpha1-antitrypsin deficiency. Am J Respir Crit Care Med 2012;185:246-59.

13. Lossau N. Röntgen verzichtete auf ein Patent: Die Welt; 2001. Available online: http://www.welt.de/print-welt/ article490586/Roentgen-verzichtete-auf-ein-Patent.html

14. Khan AN, Al-Jahdali H, Al-Ghanem S, et al. Radiology in the intensive care unit (Part 1): Normal chest radiographic appearance, instrumentaion and complications from instrumentation. Ann Thorac Med 2009;4:75.

15. Graat M, Spronk P, Schultz M. Current practice of chest radiography in critically ill patients in the Netherlands: a postal survey. Chest 2005;128:296S.

16. Rubinowitz AN, Siegel MD, Tocino I. Thoracic Imaging in the ICU. Crit Care Clin 2007;23:539-73.

17. Hill JR, Horner PE, Primack SL. ICU Imaging. Clin Chest Med 2008;29:59-76.

18. Canella C, Philippe P, Pansini V, et al. Use of Tomosynthesis for Erosion Evaluation in Rheumatoid Arthritic Hands and Wrists. Radiology 2011;258:199-205.

19. Müller NL, Coxson H. Chronic obstructive pulmonary disease $\bullet 4$ : Imaging the lungs in patients with chronic obstructive pulmonary disease. Thorax 2002;57:982-5.

20. Lynch DA, Austin JH, Hogg JC, et al. CT-Definable Subtypes of Chronic Obstructive Pulmonary Disease: A Statement of the Fleischner Society. Radiology 2015;277:192-205.

21. Bankier AA, De Maertelaer V, Keyzer C, et al. Pulmonary emphysema: subjective visual grading versus objective quantification with macroscopic morphometry and thinsection CT densitometry. Radiology 1999;211:851-8.

22. Hersh CP, Washko GR, Jacobson FL, et al. Interobserver variability in the determination of upper lobe-predominant emphysema. Chest 2007;131:424-31.

23. Muehlematter UJ, Caviezel C, Martini K, et al. Applicability of color-coded computed tomography images in lung volume reduction surgery planning. J Thorac Dis 2019;11:766-76.

24. Gevenois PA, de Maertelaer V, De Vuyst P, et al. Comparison of computed density and macroscopic morphometry in pulmonary emphysema. Am J Respir Crit Care Med 1995;152:653-7.

25. Gevenois PA, Koob MC, Jacobovitz D, et al. Whole lung sections for computed tomographic-pathologic correlations. Modified Gough-Wentworth technique. Invest Radiol 1993;28:242-6.

26. Coxson HO, Rogers RM, Whittall KP, et al. A quantification of the lung surface area in emphysema using computed tomography. Am J Respir Crit Care Med 1999;159:851-6.

27. Madani A, Van Muylem A, de Maertelaer V, et al. Pulmonary emphysema: size distribution of emphysematous spaces on multidetector CT images-comparison with macroscopic and microscopic morphometry. Radiology 2008;248:1036-41.

28. Davey C, Zoumot Z, Jordan S, et al. Bronchoscopic lung volume reduction with endobronchial valves for patients with heterogeneous emphysema and intact interlobar fissures (the BeLieVeR-HIFi study): a randomised controlled trial. Lancet 2015;386:1066-73.

29. Sciurba FC, Ernst A, Herth FJ, et al. A randomized study of endobronchial valves for advanced emphysema. N Engl J Med 2010;363:1233-44.

30. Herth FJ, Noppen M, Valipour A, et al. Efficacy predictors of lung volume reduction with Zephyr valves in a European cohort. Eur Respir J 2012;39:1334-42.

31. Mantri S, Macaraeg C, Shetty S, et al. Technical advances: measurement of collateral flow in the lung with a dedicated endobronchial catheter system. J Bronchology Interv Pulmonol 2009;16:141-4.

32. Schuhmann M, Raffy P, Yin Y, et al. Computed tomography predictors of response to endobronchial valve lung reduction treatment. Comparison with Chartis. Am J Respir Crit Care Med 2015;191:767-74.

33. Koenigkam-Santos M, Puderbach M, Gompelmann D, et al. Incomplete fissures in severe emphysematous patients 
evaluated with MDCT: incidence and interobserver agreement among radiologists and pneumologists. Eur J Radiol 2012;81:4161-6.

34. Martini K, Frauenfelder T. Emphysema and lung volume reduction: the role of radiology. J Thorac Dis 2018;10:S2719-31.

35. Ghonge NP, Chowdhury V. Minimum-intensity projection images in high-resolution computed tomography lung: Technology update. Lung India 2018;35:439-40.

36. Wang SC, Fischer KC, Slone RM, et al. Perfusion scintigraphy in the evaluation for lung volume reduction surgery: correlation with clinical outcome. Radiology 1997;205:243-8.

37. Cleverley JR, Desai SR, Wells AU, et al. Evaluation of patients undergoing lung volume reduction surgery: ancillary information available from computed tomography. Clin Radiol 2000;55:45-50.

38. Thurnheer R, Engel H, Weder W, et al. Role of lung perfusion scintigraphy in relation to chest computed tomography and pulmonary function in the evaluation of candidates for lung volume reduction surgery. Am J Respir Crit Care Med 1999;159:301-10.

39. Kauczor HU, Wielputz MO, Owsijewitsch M, et al. Computed tomographic imaging of the airways in COPD and asthma. J Thorac Imaging 2011;26:290-300.

40. Hagspiel KD, Flors L, Housseini AM, et al. Pulmonary blood volume imaging with dual-energy computed tomography: spectrum of findings. Clin Radiol 2012;67:69-77.

41. Pansini V, Remy-Jardin M, Faivre JB, et al. Assessment of lobar perfusion in smokers according to the presence and severity of emphysema: preliminary experience with dualenergy CT angiography. Eur Radiol 2009;19:2834-43.

42. Fink C, Puderbach M, Bock M, et al. Regional lung perfusion: assessment with partially parallel threedimensional MR imaging. Radiology 2004;231:175-84.

43. Ley-Zaporozhan J, Ley S, Eberhardt R, et al. Assessment of the relationship between lung parenchymal destruction and impaired pulmonary perfusion on a lobar level in patients with emphysema. Eur J Radiol 2007;63:76-83.

44. Kong X, Sheng HX, Lu GM, et al. Xenon-Enhanced Dual-Energy CT Lung Ventilation Imaging: Techniques and Clinical Applications. AJR Am J Roentgenol 2014;202:309-17.

45. Sugino K, Kobayashi M, Nakamura Y, et al. XenonEnhanced Dual-Energy CT Imaging in Combined Pulmonary Fibrosis and Emphysema. PloS one 2017;12:e0170289.
46. Lee SM, Seo JB, Hwang HJ, et al. Assessment of regional emphysema, air-trapping and Xenon-ventilation using dual-energy computed tomography in chronic obstructive pulmonary disease patients. Eur Radiol 2017;27:2818-27.

47. Chae EJ, Seo JB, Lee J, et al. Xenon ventilation imaging using dual-energy computed tomography in asthmatics: initial experience. Invest Radiol 2010;45:354-61.

48. Goo HW, Yang DH, Hong SJ, et al. Xenon ventilation CT using dual-source and dual-energy technique in children with bronchiolitis obliterans: correlation of xenon and CT density values with pulmonary function test results. Pediatr Radiol 2010;40:1490-7.

49. Park EA, Goo JM, Park SJ, et al. Collateral Ventilation Quantification Using Xenon-Enhanced Dynamic DualEnergy CT: Differences between Canine and Swine Models of Bronchial Occlusion. Korean J Radiol 2015;16:648-56.

50. Johnson KM, Fain SB, Schiebler ML, et al. Optimized 3D ultrashort echo time pulmonary MRI. Magn Reson Med 2013;70:1241-50.

51. Ohno Y, Koyama H, Yoshikawa T, et al. State-of-theArt Imaging of the Lung for Connective Tissue Disease (CTD). Curr Rheumatol Rep 2015;17:69.

52. Dournes G, Grodzki D, Macey J, et al. Quiet Submillimeter MR Imaging of the Lung Is Feasible with a PETRA Sequence at 1.5 T. Radiology 2015;276:258-65.

53. Morino S, Toba T, Araki M, et al. Noninvasive assessment of pulmonary emphysema using dynamic contrastenhanced magnetic resonance imaging. Exp Lung Res 2006;32:55-67.

54. Ley-Zaporozhan J, Puderbach M, Kauczor HU. MR for the evaluation of obstructive pulmonary disease. Magn Reson Imaging Clin N Am 2008;16:291-308, ix.

55. Puderbach M, Eichinger M, Haeselbarth J, et al. Assessment of morphological MRI for pulmonary changes in cystic fibrosis (CF) patients: comparison to thin-section CT and chest x-ray. Invest Radiol 2007;42:715-25.

56. Fuseya Y, Muro S, Sato S, et al. Complementary regional heterogeneity information from COPD patients obtained using oxygen-enhanced MRI and chest CT. PloS One 2018; 13:e0203273.

57. Martini K, Gygax CM, Benden C, et al. Volumetric dynamic oxygen-enhanced MRI (OE-MRI): comparison with CT Brody score and lung function in cystic fibrosis patients. Eur Radiol 2018;28:4037-47.

58. Zha W, Nagle SK, Cadman RV, et al. Three-dimensional Isotropic Functional Imaging of Cystic Fibrosis Using Oxygen-enhanced MRI: Comparison with Hyperpolarized 
He MRI. Radiology 2019;290:229-37.

59. Stadler A, Stiebellehner L, Jakob PM, et al. Quantitative and o enhanced MRI of the pathologic lung: findings in emphysema, fibrosis, and cystic fibrosis. Int J Biomed Imaging 2007;2007:23624.

60. Ohno Y, Hatabu H, Takenaka D, et al. Dynamic oxygenenhanced MRI reflects diffusing capacity of the lung. Magn Reson Med.2002;47:1139-44.

61. Ohno Y, Hatabu H, Murase K, et al. Quantitative assessment of regional pulmonary perfusion in the entire lung using three-dimensional ultrafast dynamic contrastenhanced magnetic resonance imaging: Preliminary experience in 40 subjects. J Magn Reson Imaging 2004;20:353-65.

62. Yilmaz E, Akkoclu A, Degirmenci B, et al. Accuracy and feasibility of dynamic contrast-enhanced 3D MR imaging in the assessment of lung perfusion: comparison with Tc-99 MAA perfusion scintigraphy. Clin Radiol 2005;60:905-13.

63. Molinari F, Fink C, Risse F, et al. Assessment of differential pulmonary blood flow using perfusion magnetic resonance imaging: comparison with radionuclide perfusion scintigraphy. Invest Radiol 2006;41:624-30.

64. Sergiacomi G, Sodani G, Fabiano S, et al. MRI lung perfusion 2D dynamic breath-hold technique in patients with severe emphysema. In Vivo 2003;17:319-24.

65. Wujcicki A, Corteville D, Materka A, et al. Perfusion and ventilation filters for Fourier-decomposition MR lung imaging. Z Med Phys 2015;25:66-76.

66. Kjørstad Å, Corteville DMR, Henzler T, et al. Noninvasive quantitative pulmonary V/Q imaging using Fourier decomposition MRI at 1.5T. Z Med Phys 2015;25:326-32.

67. Suga K, Tsukuda T, Awaya H, et al. Impaired respiratory mechanics in pulmonary emphysema: evaluation with dynamic breathing MRI. J Magn Reson Imaging 1999;10:510-20.

68. Martini K, Caviezel C, Schneiter D, et al. Dynamic magnetic resonance imaging as an outcome predictor for lung-volume reduction surgery in patients with severe emphysemadagger. Eur J Cardiothorac Surg 2019;55:446-54.

69. deSouza NM, Achten E, Alberich-Bayarri A, et al. Validated imaging biomarkers as decision-making tools in clinical trials and routine practice: current status and recommendations from the EIBALL* subcommittee of the European Society of Radiology (ESR). Insights Imaging 2019;10:87.
70. Bartholmai BJ, Raghunath S, Karwoski RA, et al. Quantitative computed tomography imaging of interstitial lung diseases. J Thorac Imaging 2013;28:298-307.

71. Raghunath S, Rajagopalan S, Karwoski RA, et al. Quantitative stratification of diffuse parenchymal lung diseases. PloS One 2014;9:e93229.

72. Occhipinti M, Paoletti M, Bartholmai BJ, et al. Spirometric assessment of emphysema presence and severity as measured by quantitative CT and CT-based radiomics in COPD. Respir Res 2019;20:101.

73. Khawaja RDA, Singh S, Madan R, et al. Ultra low-dose chest CT using filtered back projection: Comparison of $80-, 100$ - and 120\&\#xa0;kVp protocols in a prospective randomized study. Eur J Radiol 2014;83:1934-44.

74. Singh S, Kalra MK, Gilman MD, et al. Adaptive Statistical Iterative Reconstruction Technique for Radiation Dose Reduction in Chest CT: A Pilot Study. Radiology 2011;259:565-73.

75. Martini K, Barth BK, Nguyen-Kim TD, et al. Evaluation of pulmonary nodules and infection on chest CT with radiation dose equivalent to chest radiography: Prospective intra-individual comparison study to standard dose CT. Eur J Radiol 2016;85:360-5.

76. Messerli M, Ottilinger T, Warschkow R, et al. Emphysema quantification and lung volumetry in chest X-ray equivalent ultralow dose CT - Intra-individual comparison with standard dose CT.. Eur J Radiol 2017;91:1-9.

77. Zulueta JJ, Wisnivesky JP, Henschke CI, et al. Emphysema scores predict death from COPD and lung cancer. Chest 2012;141:1216-23.

78. Smith BM, Pinto L, Ezer N, et al. Emphysema detected on computed tomography and risk of lung cancer: a systematic review and meta-analysis. Lung cancer 2012;77:58-63.

79. Bergin C, Muller N, Nichols DM, et al. The diagnosis of emphysema. A computed tomographic-pathologic correlation. Am Rev Respir Dis 1986;133:541-6.

80. Foster WL, Jr., Pratt PC, Roggli VL, et al. Centrilobular emphysema: CT-pathologic correlation. Radiology 1986;159:27-32.

81. de Torres JP, Bastarrika G, Wisnivesky JP, et al. Assessing the Relationship Between Lung Cancer Risk and Emphysema Detected on Low-Dose CT of the Chest. Chest 2007;132:1932-8.

82. Wilson DO, Weissfeld JL, Balkan A, et al. Association of radiographic emphysema and airflow obstruction with lung cancer. Am J Respir Crit Care Med 2008;178:738-44.

83. Sverzellati N, Randi G, Spagnolo P, et al. Increased mean 
lung density: another independent predictor of lung cancer? Eur J Radiol 2013;82:1325-31.

84. Maldonado F, Bartholmai BJ, Swensen SJ, et al. Are Airflow Obstruction and Radiographic Evidence of

Cite this article as: Martini K, Frauenfelder T. Advances in imaging for lung emphysema. Ann Transl Med 2020;8(21):1467. doi: 10.21037/atm.2020.04.44
Emphysema Risk Factors for Lung Cancer? A Nested Case-Control Study Using Quantitative Emphysema Analysis. Chest 2010;138:1295-302. 